\title{
Les profits de la guerre. Prédation et pouvoir dans le monde franc $\left(\mathrm{VI}^{\mathrm{e}}-\mathrm{X}^{\mathrm{e}}\right.$ siècles)
}

\section{Rodolphe Keller}

\section{(2) OpenEdition}

1 Journals

Édition électronique

URL : http://journals.openedition.org/ifha/8057

DOI : $10.4000 /$ ifha. 8057

ISSN : 2198-8943

\section{Éditeur}

IFRA - Institut franco-allemand (sciences historiques et sociales)

\section{Édition imprimée}

Date de publication : 31 décembre 2014

ISSN : 2190-0078

\section{Référence électronique}

Rodolphe Keller, "Les profits de la guerre. Prédation et pouvoir dans le monde franc (VIe-Xe siècles) », Revue de l'IFHA [En ligne], 6 | 2014, mis en ligne le 31 décembre 2014, consulté le 14 novembre 2019. URL : http://journals.openedition.org/ifha/8057 ; DOI : 10.4000/ifha.8057

Ce document a été généré automatiquement le 14 novembre 2019.

(CIFHA 


\title{
Les profits de la guerre. Prédation et pouvoir dans le monde franc $\left(\mathrm{VI}^{\mathrm{e}}-\mathrm{X}^{\mathrm{e}}\right.$ siècles)
}

\author{
Rodolphe Keller
}

\section{NOTE DE L'ÉDITEUR}

Thèse de doctorat soutenue par Rodolphe Keller (EA 3350) à l'université Paris-Est/

Marne-la-Vallée, le 20 novembre 2013.

1 La prédation est un aspect important de la pratique du pouvoir dans les sociétés du haut Moyen Âge. Renvoyant à des pratiques variées - on considère ici non seulement le pillage de biens, mais également la prise de captifs, la demande de rançons ou le prélèvement de tributs - la prédation se révèle d'autant plus importante dans la société franque que la guerre y occupe une place centrale. À la fois source de richesse, pratique honorable et enjeu de pouvoir, l'appropriation de biens par la force soulève des questions de tout ordre, aussi bien économiques, que politiques et culturelles. J'ai souhaité les explorer dans cette étude, qui porte sur le rôle de la prédation dans le fonctionnement et la reproduction du pouvoir dans le monde franc, du $\mathrm{VI}^{\mathrm{e}}$ au X $\mathrm{X}^{\mathrm{e}}$ siècle. J'ai également fait le choix de me concentrer principalement sur les relations entre les Francs et les sociétés qui se situent à leurs marges orientales et septrionales, slaves et scandinaves pour l'essentiel. Cette périphérie, qui coïncide avec la frontière entre monde chrétien et sociétés païennes, est en effet le théâtre de fréquents affrontements guerriers qui se caractérisent par d'incessants transferts de richesse. C'est donc un observatoire privilégié pour cette thématique.

Ce sujet demeure très peu exploré. Dans la perspective évolutionniste qui a longtemps dominé la recherche historique, la prédation n'était rien de plus qu'un marqueur de barbarie, voire de sauvagerie, ne méritant en aucun cas d'être étudiée dans ses logiques propres. À la rigueur, les historiens se sont-ils intéressés aux conséquences que ces 
pratiques ont pu avoir pour les sociétés «civilisées » : ainsi les incursions vikings du IXe siècle étaient-elles souvent évoquées comme l'un des facteurs de la dislocation du monde carolingien. La dimension prédatrice de la société franque n'était pas, en soi, sujet d'étude. À la rigueur peut-on signaler quelques historiens du droit qui, au XIXe siècle, ont traité du pillage dans l'histoire du droit international. Ce n'est pas avant la seconde moitié du XXe siècle que quelques médiévistes commencent à mettre en avant le rôle de la prédation dans les sociétés du haut Moyen Âge, bien que les études demeurent. Georges Duby consacre quelques belles pages à ce sujet, dans un célèbre essai d'histoire économique ${ }^{1}$. L'ouverture à cette thématique doit beaucoup à l'apport de l'anthropologie, qui a contribué à ouvrir les perspectives. L'approche anthropologique est d'autant plus importante dans cette étude que la notion de prédation elle-même en est issue. Développée dans le cadre des recherches sur les sociétés indigènes d'Amérique latine, mise en avant par les anthropologues comme un aspect fondamental des relations entre les sociétés humaines ${ }^{2}$, elle permet de donner une cohérence à un segment de pratiques sociales variées, dont la spécificité est d'opérer un transfert de biens par l'usage de la force.

Une première approche a consisté à mener une étude de la terminologie utilisée par les sources pour décrire les actes de prédation, tout en précisant leur nature. À plusieurs reprises, cela a rendu nécessaire de préciser la différence entre des pratiques voisines. Ainsi le pillage (depraedatio) et le vol (rapina), clairement distincts dans le droit romain, sont moins aisés à distinguer dans des sociétés où aucun acteur n'a le monopole de l'emploi de la force. Une difficulté supplémentaire provenait du fait que le vocabulaire employé par les sources est souvent orienté : des actes de depraedatio peuvent être décrits comme de la rapina, dans le simple but d'en disqualifier les auteurs. L'étude a mis en lumière que les deux ordres de pratiques ne sont toutefois pas assimilables : la depraedatio - le pillage - est propre à une situation de conflit ouvert, à une situation déclarée d'hostilitas, ce qui n'est pas le cas du vol. Contrairement à ce dernier, elle est considérée comme légitime, pour peu qu'un rapport guerrier existe entre les acteurs. La distinction entre le don et le tribut devait aussi être précisée. Si le don se présente comme volontaire - bien que répondant, bien souvent, à une obligation - le tribut constitue une prestation ouvertement contrainte. Ici aussi, les sources se révèlent parfois ambiguës dans leur choix des mots : une même prestation opérée par un roi franc - Charles III le Gros - à un chef viking se trouve qualifiée de don par certains et de tribut par d'autres - les premiers ayant pour but de dissimuler le caractère contraint de la transaction, les seconds ayant, au contraire, clairement l'intention de mener une critique de son acte.

4 Cette étude des caractères formels des pratiques a permis ensuite, d'analyser la manière dont elles entrent en jeu dans le rapport social. La depraedatio est au cœur de la relation guerrière et c'est d'abord par rapport à celle-ci qu'elle trouve son sens. Expression matérielle du rapport d'hostilitas - comme le don l'est de l'amicitia - elle comporte une forte dimension symbolique et constitue un aspect du langage de la violence. Elle est ainsi parfois manifestement mise en œuvre dans un but de vengeance, de manière soigneusement calibrée au contexte. Si la depraedatio entre en jeu dans la modulation du rapport social, elle comporte, bien sûr, également une dimension matérielle : elle est reconnue comme une modalité légitime d'appropriation de biens et de nombreuses équipées guerrières sont motivées, avant tout, par la recherche de richesse. Face au caractère fragmentaire et succint des sources du haut Moyen Âge, il est toutefois difficile de déterminer dans quelle mesure les acteurs mènent une activité 
guerrière dans un but d'enrichissement, car les motifs s'entremêlent et sont rarement explicités. Si toute guerre comporte une forte dimension prédatrice, presque aucune ne se résume uniquement à cela. Dans quelques rares cas seulement, il est possible de percevoir sans ambiguïtés le tropisme prédateur qui oriente les choix des acteurs.

Le prélèvement de tributs - qu'il s'agisse des tributs imposés par les Vikings aux Francs dans le cadre de leurs incursions, ou de ceux prélevés par les Francs sur leurs voisins, repose également sur la contrainte mais relève de mécanismes différents. Il implique des processus de négociations et de médiation. Il s'accompagne parfois même de phénomènes d'intégration, comme le montre le cas de ces chefs vikings qui, après avoir reçu un tribut des reges carolingiens, viennent intégrer la haute société franque. Cette étude s'est surtout concentrée sur le régime tributaire imposé par les Francs aux peuples périphériques. La gens tributaria est soumise à un rapport de domination que les sources assimilent à celui qui s'exerce sur le servus au sein de la société franque. Cette forme de domination permet aussi bien d'obtenir des revenus réguliers - qui bénéficient directement à l'institution royale -, que de sécuriser l'espace frontalier. Dès le VIe siècle, Clovis et ses successeurs imposent ainsi un statut de tributaire aux Saxons, aux Gascons ou encore aux Lombards. L'expansion carolingienne du VIII siècle amène à une transformation du système tributaire : l'intégration des Saxons et de plusieurs principautés périphériques dans le royaume conduit Charlemagne à imposer un régime tributaire sur les Slaves, ce qui est d'abord un moyen de stabiliser l'espace oriental. La Francie orientale du IXe siècle et la Germanie ottonienne du Xe siècle héritent de ce système. Si le régime tributaire est un moyen, pour la royauté franque, d'instaurer un contrôle sur des groupes sans procéder à leur intégration dans le royaume et sans intervenir directement dans leur organisation, il est probable qu'il a pour conséquence des transformations locales qui nous échappent largement, les sources écrites faisant défaut. Certains acteurs locaux se montrent disposés à accueillir la domination franque, ce qui suggère qu'ils jouent un rôle dans l'organisation locale du prélèvement tributaire, voire qu'ils y trouvent un avantage. La domination tributaire s'accompagne ainsi de processus de concentration du pouvoir qui bénéficient aux interlocuteurs du pouvoir franc.

6 Pillages, tributs : tout cela est source de richesses qu'il est impossible de quantifier précisément. En dehors de quelques cas exceptionnels, les bénéfices qu'en tirent les acteurs particuliers nous sont inconnus et seul quelques remarques générales peuvent être faites. Des biens de toute nature sont appropriés, allant des moyens de subsistance aux objets précieux. Les sources insistent surtout sur les biens de valeurprincipalement en argent ou en or -, et souvent sur les armes, comme les épées et les chevaux, des biens qui sont essentiels dans la praxis aristocratique. Les tributs donnent lieu - plus souvent que le pillage - à quelques indications chiffrées. On constate que les écarts sont très importants : les tributs en nature, donnés par les peuples périphériques septentrionaux et orientaux sont négligeables par rapport aux tributs en solidi d'or versés par les peuples italiens. Les tributs exigés sont ainsi définis par le pouvoir franc en fonction des structures économiques des gentes concernées. De cette étude des biens, il est ressorti surtout que - au-delà des quantités concernées - la prédation se révèle d'abord comme un moyen d'obtenir des choses qu'il est difficile d'obtenir autrement, soit parce qu'elles ne circulent pas, soit parce qu'elles le font selon des modalités autres que l'échange commercial. Dans certains contextes, cela semble être le cas des armes, que l'on voit surtout circuler par l'intermédiaire de prestations obligatoires, ainsi que par la pratique du don et de la prédation. L'appropriation contrainte de certains biens 
constitue ainsi un enjeu particulier, et cela d'autant plus qu'elle est pleinement intégrée à l'habitus aristocratique : prendre par la force des objets prestigieux investit celui qui s'en approprie d'un surcroît de capital symbolique. À l'époque mérovingienne, on l'observe de manière frappante dans les cas fréquents d'appropriation du trésor, incarnation du pouvoir du roi.

7 L'utilisation qui est faite des richesses ainsi acquises est également difficile à cerner. Les sources donnent des informations uniquement lorsque ces biens sont employés immédiatement après leur appropriation - partagés ou distribués. On entrevoit ainsi quelques mécanismes de l'usage qui est fait du butin au sein des groupes armés. Il est un élément important de la cohésion guerrière. Les normes qui organisent sa répartition sont étroitement liées à la nature des relations qui unissent les groupes et la mise en cause des règles peut être source de tensions, voire de ruptures. Le partage entre les guerriers - que l'on voit à l'œuvre dans l'anecdote du vase de Soissons, rapportée par Grégoire de Tours - est associé à des relations à caractère horizontal, alors que les différentes formes de distribution du butin par un chef de guerre expriment le rapport vertical qui existe entre lui et les guerriers. La logique du partage s'observe surtout à l'époque mérovingienne: son respect est la garantie, pour les potentes, de pouvoir mobiliser des paysans guerriers qui ne reçoivent pas de solde. Dès le VIIe siècle, avec la disparition des levées générales ou locales, ces mécanismes deviennent marginaux. Au sein des armées des grands, composées principalement de leurs clientèles, les pratiques sont différentes. Le butin est probablement employé dans une certaine mesure à l'entretien de la suite des grands, mais ce mécanisme nous échappe largement. Il est en revanche certain que le chef de guerre distribue parfois le butin aux fidèles qui sont présents lors des opérations guerrières. Si ces distributions sont faites à sa discrétion, il a tout intérêt à en effectuer ponctuellement, afin de renforcer les fidélités et la cohésion de son groupe. Dans le contexte de l'ascension des Pippinides au VIIIe siècle, ce principe de la distribution devient la norme au sein de l'armée des Francs.

8 En dehors des groupes armés, le butin fait parfois l'objet de pratiques ostentatoires illustrant la victoire des chefs de guerre. Il peut être donné, ce qui permet aussi bien de renforcer les fidélités que de manifester la largitas d'un prince. Seules les pratiques les plus spectaculaires nous sont connues. Leur sens est étroitement lié au contexte sociopolitique. Clovis effectue à plusieurs reprises des dons du butin aux églises, en guise de réparation pour les déprédations qu'elles ont subies. En 508 il fait bénéficier la basilique Saint-Martin du butin fait contre les Wisigoths ariens, après quoi, au cours d'une parade triomphale dans la ville de Tours, il en distribue une part au peuple présent, manière d'associer l'abbaye et la ville à la construction du royaume. Au cours du VIII ${ }^{e}$ siècle, la documentation devient plus abondante et montre que la pratique triomphale se maintient. Pendant le règne de Charlemagne, on observe à de nombreuses reprises une pratique spécifique : la présentation du butin par un chef de guerre au roi. La fréquence de cette pratique à ce moment s'explique peut-être par une exacerbation de la compétition entre les élites, qui amène à multiplier les pratiques ostentatoires. Le don du trésor avar par le duc Éric de Frioul à Charlemagne, en 795, en est l'exemple le plus frappant, en même temps qu'il marque le début d'une pratique qui fait, en quelques années, de nombreux émules. La distribution de ce même butin avar par Charlemagne aux grands du monde franc est à la mesure du trésor qui lui a été livré par Éric de Frioul et soutient - comme au temps de Clovis - un projet politique. Cette distribution vient appuyer la prétention œcuménique de la royauté carolingienne : par 
le don au pape, aux rois et aux églises anglo-saxonnes, Charlemagne manifeste sa puissance tout autant qu'il se présente comme le protecteur de la chrétienté. Par la même occasion, il dessine finement les contours du populus christianus sur lequel porte sa prétention: Byzance n'est pas concernée par cette commune participation aux bénéfices de la guerre contre les Avars.

Un dernier chapitre de cette thèse vise à préciser comment la prédation entre en jeu dans les équilibres des différents moments du royaume franc. À l'époque mérovingienne, la prédation caractérise surtout les relations conflictuelles au sein des tria regna et de leurs dépendances, plus qu'elle ne mobilise les Francs dans leur ensemble contre les ennemis extérieurs. Sa pratique fréquente s'explique par l'importance toute particulière des biens mobiliers comme assise du pouvoir et comme outil de construction des relations sociopolitiques. Lors de l'ascension des Pippinides, on observe deux inflexions. D'une part, à mesure que les maires du palais mobilisent les élites dans une dynamique expansionniste, la prédation se concentre davantage contre les ennemis des Francs. Parallèlement, l'évolution des structures sociales - en particulier l'usage croissant de ressources foncières dans la construction des fidélités militaires - réduit la dépendance des potentes à l'égard de la prédation de biens mobiliers. Cette évolution est renforcée par l'intégration des gentes périphériques au royaume et par l'accroissement des ressources foncières disponibles. Au IXe siècle, avec la fin de la dynamique expansionniste, ce mécanisme se grippe: après 843, un déséquilibre croissant oppose la Francie occidentale, où le tropisme prédateur ne semble pas absent des troubles qui touchent le royaume, à la Francie orientale de Louis le Germanique et de ses successeurs, où la royauté conserve la possibilité de canaliser l'activité guerrière des grands vers l'extérieur du royaume, principalement les frontières slaves, qui font figure d'espace privilégié de la compétition entre les grands. À l'époque ottonienne, le régime tributaire sous lequel sont placés les peuples slaves évolue vers un régime de gestion directe du territoire, qui amène les acteurs du pouvoir à rivaliser pour la domination locale. La lutte pour les honores, pour le partage des tributs et des ressources foncières se traduit par des conflits qui troublent fortement l'espace slave. Celui-ci demeure peu encadré par la royauté et certains acteurs en rupture y trouvent un contexte favorable pour s'installer et développer leurs assises, ce qui contribue à reconfigurer les hiérarchies sociopolitiques.

Cette étude de la prédation dans le monde franc a ainsi permis d'éclairer sous un angle nouveau de nombreux aspects de l'exercice du pouvoir, tout en ouvrant un certain nombre de perspectives. Un axe qui mériterait tout particulièrement d'être approfondi, est celui des modalités de circulation des biens. Certaines classes de biens font à l'évidence l'objet d'une circulation restreinte et échappent - du moins en partie - aux circuits de l'échange commercial, d'où l'importance de la prédation. Plusieurs remarques ont déjà été faites à propos des armes et d'autres pourraient sans doute être faites sur l'ensemble des biens précieux. Il conviendra à l'avenir de mobiliser l'ensemble de la documentation disponible - faisant également davantage place à l'archéologie - afin de préciser la manière dont les biens circulent et les enjeux qui portent sur leur accessibilité, ce qui peut permettre de mieux comprendre les stratégies des acteurs. Certains potentes en rupture multiplient parfois les actes de depraedatio contre leur environnement proche, ce qui pourrait d'autant mieux s'expliquer qu'ils sont exclus - du moins temporairement - des réseaux de l'échange. Afin d'avoir une compréhension précise de ces phénomènes, il nous faut ainsi considérer d'un seul mouvement les conditions économiques et la manière dont les acteurs agissent et 
interagissent dans un contexte donné. De ce point de vue, l'enjeu est moins de considérer la prédation pour elle-même, que de repenser le rapport des élites à la richesse en intégrant ce paramètre.

\section{NOTES}

1. Georges Duby, Guerriers et paysans, VII ${ }^{-}$XII ${ }^{e}$ siècles. Premier essor de l'économie européenne, Paris, 1973, p. 61-69. Signalons aussi : Philip H. Grierson, «Commerce in the Dark Ages : a Critique of the Evidence ", dans Transactions of the Royal Historical Society, 5/9 (1959), p. 123-140 et Timothy REUTER, «Plunder and Tribute in the Carolingian Empire », dans Transactions of the Royal Historical Society, 5/35 (1985), p. 75-94.

2. Philippe Descola, Par-delà nature et culture, Paris, 2005, p. 426-439.

\section{AUTEUR}

\section{RODOLPHE KELLER}

(EA 3350, Paris) 